\title{
Preparation and Complex Characterization of Magnetic Nanoparticles in Magnetic Fluid
}

\author{
M. Kubovčíková, I. Antal, J. Kováč, V. ZÁvišová, M. Koneracká*, P. KopČanskÝ \\ Institute of Experimental Physics SAS, Watsonova 47, 04001 Košice, Slovakia
}

\begin{abstract}
This paper deals with the preparation and complex characterization of magnetite nanoparticles (MNPs), stabilized with sodium oleate (SO), by the routine methods such as infrared spectroscopy (FTIR), magnetic measurements, scanning electron microscopy (SEM) and dynamic light scattering (DLS). The FTIR spectra showed that SO molecules were linked to MNPs through chemical bonding. Magnetic measurements proved that the MNPs are superparamagnetic in nature. Four different methods were used to determine the size and size distribution of the MNPs: SEM, DLS, differential centrifugal sedimentation (DCS) and magnetic measurements. SEM analysis showed a relatively narrow size distribution of roughly spherical MNPs with a mean diameter of $61 \mathrm{~nm}$. DLS analysis confirmed monodispersed MNPs production with hydrodynamic diameter of $75 \mathrm{~nm}$. The size distribution determined by DCS was found to be $69 \mathrm{~nm}$. Finally, the calculated magnetic core diameter obtained from magnetization curve was $10 \mathrm{~nm}$. The obtained results demonstrate that SO coated MNPs fulfil the requirements for a useful drug delivery system.
\end{abstract}

DOI: 10.12693 /APhysPolA.126.268

PACS: 75.75.Fk, 78.67.Bf, 36.40.Cg, 47.65.Cb, 75.50.Mm, 75.30.Cr

\section{Introduction}

In the past few years, superparamagnetic MNPs characterized by higher magnetization and good biocompatibility have attracted significant attention as magnetic drug-targeting carriers and for controlled drug release and also for hyperthermia. Surface modified superparamagnetic MNPs, characterized by the absence of magnetism on the removal of the magnetic field, can be intravenously delivered to the tumour site using an external magnetic field. However, superparamagnetic MNPs which are not surface modified with a large surface areato-volume ratio tend to agglomerate and form large clusters, with the consequent loss of their superparamagnetic characteristics. An important disadvantage of these magnetic particles for drug delivery systems is that they are rapidly cleared by macrophages or the reticuloendothelial system before they are able to reach the site of the tumour cell.

In this paper we focus mainly on the preparation and full characterization of superparamagnetic MNPs stabilised by $\mathrm{SO}$, with the aim to prepare stable magnetic fluid (MF). Further MF modification with specific biocompatible material could offer a high potential for biomedical application.

\section{Experiment}

Magnetite nanoparticles were synthesized by coprecipitation of iron (II) and (III) and coated with sodium oleate. For preparation of magnetite nanoparticles, $2.10 \mathrm{~g}$ $\mathrm{FeCl}_{3} \times 6 \mathrm{H}_{2} \mathrm{O}$ and $1.1 \mathrm{~g} \mathrm{FeSO}_{4} \times 7 \mathrm{H}_{2} \mathrm{O}$ were dissolved in $40 \mathrm{ml}$ distilled water. Under vigorous stirring, $10 \mathrm{ml} 25 \%$ ammonium hydroxide solution was added into the flask, and magnetite in the form of a black precipitate formed

*corresponding author; e-mail: konerack@saske.sk immediately. After washing the particles were isolated from water and $0.75 \mathrm{~g}$ of the surfactant sodium oleate was added. This mixture was stirred well and heated at $80{ }^{\circ} \mathrm{C}$ for $1 \mathrm{~h}$. Agglomerates were removed by centrifugation at $9000 \mathrm{rpm}$ for $30 \mathrm{~min}$.

FTIR spectroscopy was used to confirm adsorption of SO molecules on MNPs surface. SEM gives detailed shape and morphological information, DLS and DCS enable a description of the particle size, size distribution and polydispersity. DLS evaluates the intensity fluctuation of scattered light reflected from MNPs in suspension. The fluctuation is resulting from the "Brownian motion" that keeps the particles in steady movement. The particle size measurements by DLS were carried out using a Malvern Zetasizer NanoZS. DCS determines particle size by measuring the time required for the colloidal particles to settle in a density gradient in a disc centrifuge. The DC24000 UHR disc centrifuge (CPS Instruments, Inc.) was used to perform sedimentation based size distribution measurements.

In order to verify the superparamagnetic behaviour of MNPs and to monitor colloidal stability of MF, a Quanttum Design MPMS XL-5 SQUID magnetometer was exploited.

\section{Results and discussion}

The morphology of SO coated magnetic nanoparticles is shown in Fig. 1. It can be seen that the particles are nearly spherical and their average diameter is about $61 \mathrm{~nm}$.

The FTIR spectra of pure magnetite and SO coated magnetite were recorded between 4000 and $500 \mathrm{~cm}^{-1}$ and showed that SO molecules were linked to MNPs through chemical bonding (data are not shown).

The temperature dependences of magnetization curves (ZFC and FC) were measured at 100 Oe for both samples and a blocking temperature $T_{B}$ was determined according to method described in [1]. 


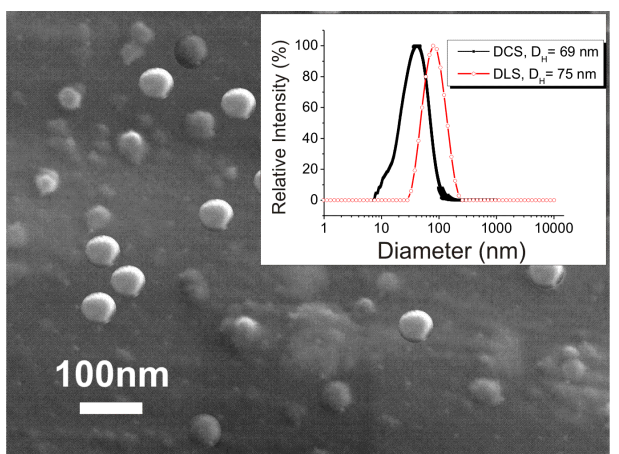

Fig. 1. SEM image of SO coated MNPs. (Inset: DLS and DCS size distribution of SO coated MNPs).

When the MNPs are chemically coated with SO, as shown in Fig. 2, the $T_{B}$ is suppressed to a lower temperature [2]. Without coating of surfactant on the particles, due to the increase in the large ratio of surface area to the volume, the attractive force between the nanoparticles will increase, and agglomeration of the nanoparticles will take place. These agglomerated MNPs act as a cluster, resulting in an increase of the blocking temperature. In contrast, in the SO coated MNPs the surfactant molecules bonded to single particles prevent them from agglomeration. The magnetization curves of the MNPs as well as SO coated MNPs, measured at room temperature, showed the typical characteristics of superparamagnetic behaviours (data are not shown). The measured saturation magnetization for the uncoated MNPs was found to be $66 \mathrm{~A} \cdot \mathrm{m}^{2} / \mathrm{kg}$ at $280 \mathrm{~K}$ and for SO coated $\mathrm{MNP}_{S} 28.1 \mathrm{~A} \cdot \mathrm{m}^{2} / \mathrm{kg}$.

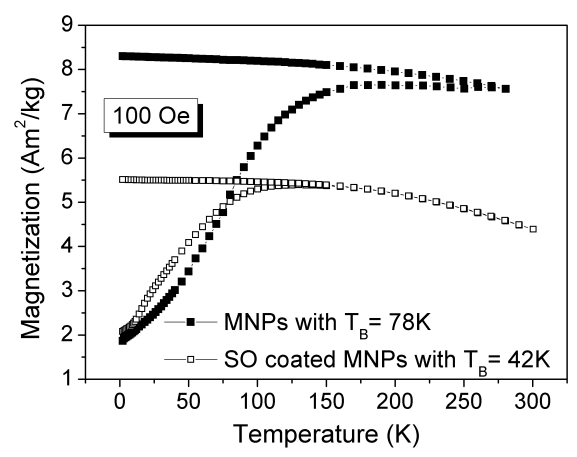

Fig. 2. ZFC and FC curves for pure MNPs and SO coated MNPs.

To monitor colloidal stability of MNPs, the temperature-induced transitions of SO coated MNPs were studied through a combination of DLS and AC susceptibility measurements. As presented in Fig. 3, no sharp change in hydrodynamic diameter as well as in the high frequency susceptibility (Fig. 3) was observed, indicating sample colloidal stability [3].

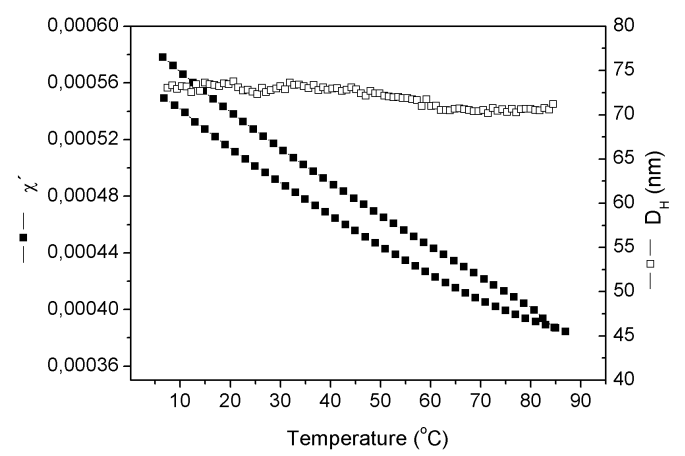

Fig. 3. Temperature response of the AC susceptibility and DLS measurement of the $\mathrm{D}_{H}$ as a function of temperature for SO coated MNPs.

As the size and size distribution of MNPs to be used as drug delivery system are important parameters, several different methods were used in this work to determine the size and size distribution of the MNPs: SEM, DLS, DCS and magnetic measurements. SEM analysis showed a relatively narrow size distribution of roughly spherical MNPs with a mean diameter of $61 \mathrm{~nm}$. DLS analysis confirmed monodispersed MNPs production with hydrodynamic diameter $D_{H}$ of $75 \mathrm{~nm}$. The size distribution determined by DCS using sedimentation velocity analysis was found to be $69 \mathrm{~nm}$. Finally, the calculated magnetic core diameter obtained from magnetization curve using Langevin function [4] was $10 \mathrm{~nm}$. While SEM, DLS and DCS gives the hydrodynamic diameter of particles (nonmagnetic layer along with magnetic core), diameter resulting from magnetic measurements depends only on magnetic moment of MNPs and no effect from nonmagnetic layer is involved.

Considering the meaning of the diameter values, the agreement between the methods is very good and the data demonstrate that SO coated MNPs fulfil the requirements for a useful drug delivery system.

\section{Acknowledgments}

This work was supported within the projects 26220120021 in the frame of Structural Funds of EU, VEGA 2/0041/12, 2/0045/12 and 1/0861/12, Slovak Research and Development Agency under the contracts No. APVV-0742-10, APVV-0171-10.

\section{References}

[1] J.J. Lu, H.Y. Deng, H.L. Huang, J. Magn. Magn. Mater. 209, 37 (2000).

[2] D.K. Kim, Y. Zhang, W. Voit, K.V. Rao, M. Muhammed, J. Magn. Magn. Mater. 225, 30 (2001).

[3] A.P. Herrera, C. Barrera, Y. Zayas, C. Rinaldi, J. Colloid Interface Sci. 342, 540 (2010).

[4] S. Yoon, J. Magnetics 16, 368 (2011). 